\title{
Anticancer evaluation of di- and trifunctional substituted 1,3-thiazoles
}

\author{
Kostyantyn V. Turov, Oleg P. Mitiukhin, Svitlana A. Chumachenko, Vladimir S. Zyabrev, \\ Volodymyr S. Brovarets*
}

V. P. Kukhar Institute of Bioorganic Chemistry and Petrochemistry of the NAS of Ukraine, 1 Murmanska St., Kyiv, 02094, Ukraine

\begin{abstract}
Anticancer activity of a series of polyfunctional substituted 1,3-thiazoles has been studied within the international scientific program "NCI-60 Human Tumor Cell Lines Screen". Screening was performed in vitro on 60 cell lines of lungs, kidneys, CNS, ovaries, prostate, and breast cancer, epithelial cancer, leukemia, and melanoma. The most effective compounds were those with a piperazine substituent at $C^{2}$ of the 1,3-thiazole cycle: 1-(4-((4-methylphenyl)sulfonyl)-2-phenyl-1,3-thiazol-5-yl)piperazine (average lg GI50 = -5.87, $\lg$ TGI $=-5.54, \lg$ LC $\left._{50}=-5.21\right), 1-(2-(3,5$-dimethyl-1H-pyrazol-1-yl)-4-((4-methylphenyl)sulfonyl)-1,3-thiazol-5-yl)piperazine (average $\left.\lg \mathrm{GI}_{50}=-5.66, \lg \mathrm{TGI}=-5.26, \lg \mathrm{LC}_{50}=-4.83\right)$, and 1-(2,4-bis((4-methylphenyl)sulfonyl)-1,3-thiazol-5-yl)piperazine (average lg GI $50=$ $\left.-5.67, \lg \mathrm{TGI}=-5.21, \lg \mathrm{LC}_{50}=-4.67\right)$.
\end{abstract}

Keywords: 1,3-thiazole; anticancer activity; growth inhibitor; cytostatic activity; cytotoxic activity.

\section{Introduction}

Derivatives of 1,3-thiazoles play an important role in basic and applied research. It has been demonstrated that 1,3-thiazoles are widely used for creation of dyes, insecticides, herbicides, and pharmaceuticals. Di- and trisubstituted 1,3-thiazole are effective anti-inflammatory, anthelmintic, antiviral, and bactericidal agents [1-6]. The nature of the chemical groups in the heterocycle can significantly affect their pharmacological properties. Despite of wide range of thiazole libraries, many of trisubstituted thiazoles stays unavailable due to multistep and complicated pathways for its synthesis.

The purpose of this work was to synthesize and evaluate the antitumor activity of di- and tri- substituted 1,3-thiazole. $\mathrm{S}_{\mathrm{N}} \mathrm{Ar}$ reactions were convenient for direct introduction of substituents into proper positions of heterocycle. Well known that substitution of halogen atom in $\mathrm{C}^{4}$ or $\mathrm{C}^{5}$ position demands high temperatures and $\mathrm{Pd}$ catalysis.

\begin{tabular}{ll}
\hline Received: & 20.03 .2020 \\
Revised: & 03.04 .2020 \\
Accepted: & 10.04 .2020 \\
Published online: & 30.06 .2020 \\
\hline
\end{tabular}

* Corresponding author. Tel.: +380-44-573-2596; e-mail: brovarets@bpci.kiev.ua (V.S. Brovarets) ORCID: 0000-0001-6668-3412
Present of EWG makes liable of halogen atom in position 5 of thiazole ring. That allows to modify one by introducing different $\mathrm{O}, \mathrm{N}$ and substituents. Such reactions pass in mild conditions and with high level of regioselectivity, that's why yields of desired products was pretty fine.

\section{Results and Discussion}

\section{Chemistry}

Syntheses of compounds $\mathbf{1 - 2 3}$ are presented in Schemes 1 and 2. 1-R-3-Tosyl-1,4,4-trichloro-2-aza-1,3butadienes I and 1-tosyl-2,2-dichloroethenylisothiocyanate II were used as starting compounds. Imidoyl chlorides I react with thiourea to give 2-R-4-tosyl-5-chloro-1,3thiazoles 1, 10. 5-Chloro-1,3-thiazole 1 reacts with $\mathrm{N}$-, $\mathrm{O}$ and $S$-nucleophiles to eliminate the chlorine anion and form the corresponding 1,3-thiazole derivatives 2-7. Heating of compound $\mathbf{3}$ with hydrogen peroxide in acetic acid followed by the reaction of the obtained product with morpholine or benzylamine yields 4-aminosubstituted 5-((4-chlorophenyl) sulfanyl)-2-phenyl-1,3-thiazoles 8, 9.

1-Tosyl-2,2-dichloroethenylisothiocyanate II was used for the synthesis of trifunctionally substituted 1,3-thiazoles 11-23. Compound II was treated with thiophenols or alkyl mercaptans in the presence of pyridine. A cyclization took place to form intermediate 2-aryl(alkyl)sulfanyl-4-tosyl-5-

(C) Turov K. V. et al. This is an open-access article distributed under the terms of the Creative Commons Attribution License, which permits unrestricted use, distribution, and reproduction in any medium, provided the original author and source are credited. 


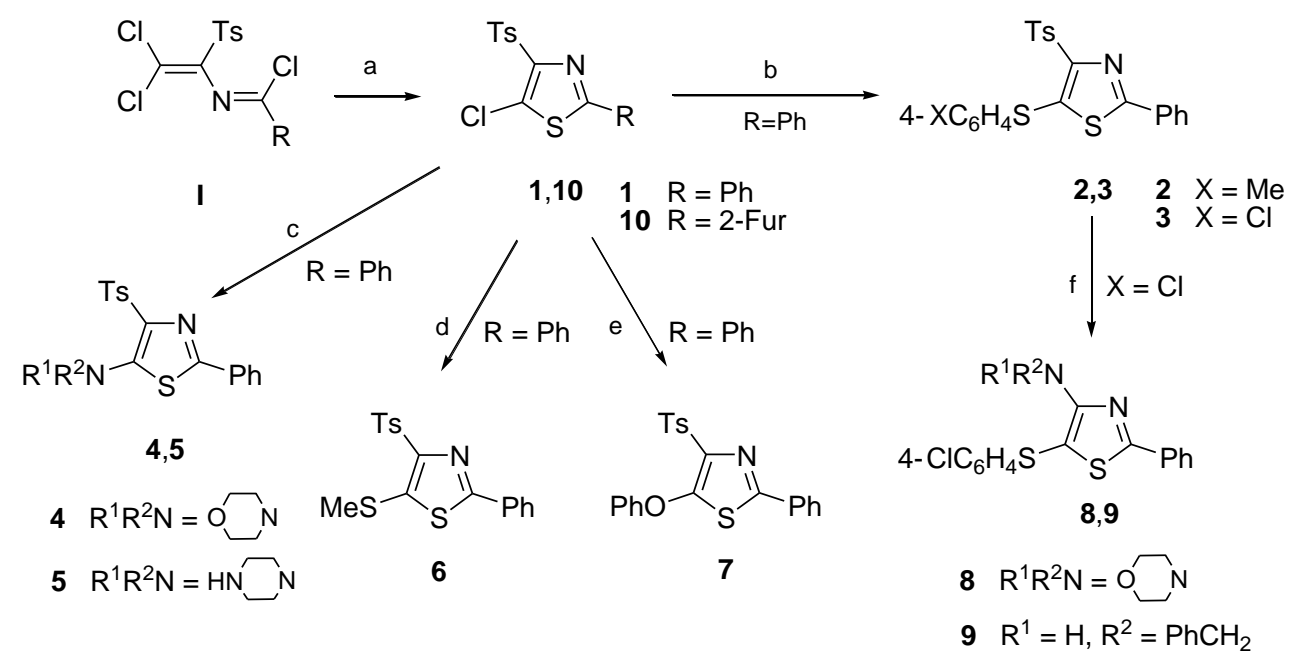

Scheme 1. Synthesis of 4,5-difunctional substituted 1,3-thiazoles 1-10. Reagents and conditions: (a) $\left(\mathrm{H}_{2} \mathrm{~N}\right)_{2} \mathrm{C}=\mathrm{S}$ (excess), MeCN, reflux, $2 \mathrm{~h}$; (b) 4- $\mathrm{MeC}_{6} \mathrm{H}_{4} \mathrm{SH}$ or $4-\mathrm{ClC}_{6} \mathrm{H}_{4} \mathrm{SH}, \mathrm{Et} 3 \mathrm{~N}$, MeCN, reflux, $2 \mathrm{~h}$; (c) morpholine or piperazine (excess), dioxane, $100{ }^{\circ} \mathrm{C}, 24 \mathrm{~h}$; (d) $\mathrm{NaSH}$ (excess), THF, $60{ }^{\circ} \mathrm{C}, 3 \mathrm{~h}$; MeI, MeONa, MeOH, $20{ }^{\circ} \mathrm{C}$, $5 \mathrm{~h}$; (e) PhONa, THF, $20{ }^{\circ} \mathrm{C}, 24 \mathrm{~h}$; (f) $\mathrm{H}_{2} \mathrm{O}_{2}$ (excess), $\mathrm{CF}_{3} \mathrm{CO}_{2} \mathrm{H}$, reflux, 3 h; morpholine or $\mathrm{PhCH}_{2} \mathrm{NH}_{2}$ (excess), dioxane, $100^{\circ} \mathrm{C}, 24 \mathrm{~h}$.

chloro-1,3-thiazoles, which were oxidized with hydrogen peroxide to 1,3-thiazoles III containing at position $\mathrm{C}^{2}$ an arylsulfonyl or an alkylsulfonyl and at position $\mathrm{C}^{4}$ the tosyl group. Substitution of the chlorine atom at position $\mathrm{C}^{5}$ of compounds III with 4-chlorothiophenol followed by the oxidation of the sulfanyl group under the action of hydrogen peroxide in trifluoroacetic acid yields 1,3-thiazole derivatives IV with three different sulfonyl groups. 1,3-Thiazoles 11-16 were obtained under the treatment of compounds IV by $N$ - and $O$-nucleophiles. The reaction of 1,3-thiazoles IV with dimethylamine, benzylamine, or ammonia in a molar ratio of $1: 2$ at $20^{\circ} \mathrm{C}$ yields 2 -amino1,3-thiazole derivatives $\mathbf{1 1 - 1 3}$ as a result of replacing a sulfonyl group at position $\mathrm{C}^{2}$. Treatment of thiazoles IV with the excess of an amine or sodium 4-chlorophenolate, leads to the substitution of the two arylsulfonyl groups yielding compounds 14-16. 1,3-Thiazole 17 was obtained from reagent II, hydrazine hydrate, and acetylacetone. It gives when heated with morpholine or piperazine, the corresponding 5-amino-2-pyrazolyl-4-tosyl-1,3-thiazoles 18, 19 and, when treated with sodium hydrogen sulfide followed by propyl iodide, 1,3- thiazole $2 \mathbf{2 0}$

5-Piperazino-substituted 1,3-thiazoles 21, 22 were prepared by the nucleophilic substitution of the chlorine atom in compounds III for piperazine in boiling ethanol. 1,3-Thiazole $\mathbf{2 3}$ was obtained from isothiocyanate II, methyl mercaptan, piperazine, and hydrochloric acid.

Structures of synthesized compounds shown in Table 1 were confirmed by ${ }^{1} \mathrm{H}$ NMR spectra and elemental analysis.

\section{Biological Evaluation}

Anticancer activity of the synthesized compounds was studied within an international scientific program of the US National Institutes of Health. The screening was performed in vitro on 60 cell lines of lungs, kidneys, CNS, ovaries, prostate, and breast cancer, epithelial cancer, leukemia, and melanoma at a substance concentration of $10^{-5} \mathrm{M}$. Growth percentage (GP) of cancer cells compared to the control (in the absence of a chemical substance, 100\%) was determined [7-10]. Synthesized 1,3-thiazole derivatives have been shown to be active against several types of cancer cells (Table 1).

For example, 2-phenyl-4-tosyl-5-chloro-1,3-thiazole (1) considerably inhibits growth of cells of leukemia (K-562, $\mathrm{GP}=30.07 \%$ and $\mathrm{SR}, \mathrm{GP}=13.60 \%$ ), lung cancer (NCI$\mathrm{H} 522, \mathrm{GP}=44.91 \%)$, melanoma $(\mathrm{M} 14, \mathrm{GP}=37.06 \%$ and MDA-MB-435, GP = 1.37\%), and breast cancer (MDAMB-468, GP $=0.73 \%$ ).

The replacement of the chlorine atom in compound $\mathbf{1}$ with p-tolylsulfanyl group (compound 2) results in a significant $70 \%$ reduction of the inhibitory activity towards leukemia and lung cancer cells and in the full extinction of the activity towards melanoma cells MDA-MB-435 $(\mathrm{GP}=106.38 \%)$ and M14 (GP = 102.19\%).

The substitution of the chlorine atom by methylsulfanyl group (compound 6) results in a uniform decrease of the inhibitory activity towards leukemia and lung cancer cells and does not change melanoma and breast cancer cells inhibition. The substitution of the chlorine atom in compound 1 with morpholine (compound 4) as well as phenoxy group (compound 7) also does not change leukemia and melanoma cells growth inhibition. In summary, any replacement of the chlorine atom results in a decrease of inhibitory activity in relation to parent compound 1 .

1,3-Thiazoles 8,9 containing the 4-chlorophenylsulfonyl group at position $\mathrm{C}^{5}$ and a substituted amino group at position $\mathrm{C}^{4}$ are somewhat more active than compound 4 with the morpholino group at position $\mathrm{C}^{5}$. Thus, 1,3-thiazole 


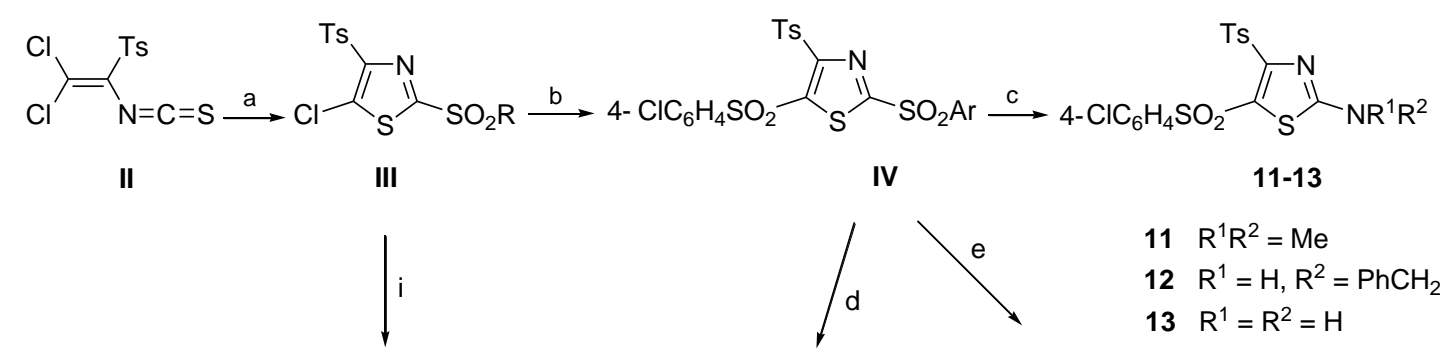<smiles>O=POc1nc(S)c(N2CCNCC2)s1</smiles>

21,22<smiles>CCCCOc1sc(NPCC)nc1N=P</smiles>

14,15

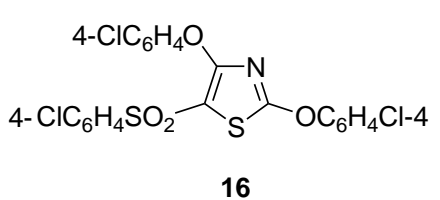

16

$$
\begin{aligned}
& 21 \mathrm{R}=4-\mathrm{MeC}_{6} \mathrm{H}_{4} \\
& 22 \mathrm{R}=\mathrm{Pr}
\end{aligned}
$$

$$
\begin{array}{ll}
14 & R^{1}=R^{2}=\left(\mathrm{CH}_{2}\right)_{4} \\
15 & R^{1}=H, R^{2}=\mathrm{PhCH}_{2}
\end{array}
$$<smiles>CCCNc1sc(-n2nc([As])cc2C)nc1C</smiles>

18,19<smiles>[3H]c1nc(-n2nc(C)cc2C)sc1Cl</smiles>

17<smiles>CO[SH](C)(=O)c1nc([As])c(N2CCNCC2)s1</smiles>

23

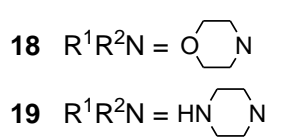<smiles>Cc1cc(C)n(-c2nc(S)c(S)s2)n1</smiles>

20

Scheme 2. Synthesis of 2,4,5-trifunctional substituted 1,3-thiazoles 11-23. Reagents and conditions: (a) ArSH or PrSH, Py, benzene, $15{ }^{\circ} \mathrm{C}, 8 \mathrm{~h} ; \mathrm{H}_{2} \mathrm{O}_{2}$ (excess), AcOH, reflux, $4 \mathrm{~h}$; (b) $4-\mathrm{ClC}_{6} \mathrm{H}_{4} \mathrm{SH}, \mathrm{Et} 3 \mathrm{~N}$, THF, $5{ }^{\circ} \mathrm{C}, 30 \mathrm{~h} ; \mathrm{H}_{2} \mathrm{O}_{2}$ (excess), $\mathrm{CF}_{3} \mathrm{CO}_{2} \mathrm{H}$, reflux $4 \mathrm{~h}$; (c) $\mathrm{Me}_{2} \mathrm{NH}_{\text {or }}$ $\mathrm{PhCH}_{2} \mathrm{NH}_{2}$, or $\mathrm{NH}_{3}$, THF, $20{ }^{\circ} \mathrm{C}, 24 \mathrm{~h}$; (d) piperidine or $\mathrm{PhCH}_{2} \mathrm{NH}_{2}$ (excess), THF, $60{ }^{\circ} \mathrm{C}, 48 \mathrm{~h}$; (e) $4-\mathrm{ClC}_{6} \mathrm{H}_{4} \mathrm{ONa}$ (excess), THF, $20{ }^{\circ} \mathrm{C}$, $24 \mathrm{~h}$; (f) $\mathrm{NH}_{2} \mathrm{NH}_{2} \mathrm{H}_{2} \mathrm{O}$ (excess), THF, $20{ }^{\circ} \mathrm{C}, 5 \mathrm{~h} ; \mathrm{Ac}_{2} \mathrm{CH}_{2}$ (excess), $\mathrm{AcOH}$, reflux, $10 \mathrm{~h}$; (g) morpholine or piperazine (excess), BuOH, reflux, $20 \mathrm{~h}$; (h) $\mathrm{NaSH}$ (excess), $\mathrm{MeOH}, 20-25^{\circ} \mathrm{C}, 20 \mathrm{~h}$; PrI, MeONa, MeOH, reflux, 3 h; (i) piperazine (excess), EtOH, reflux, $1 \mathrm{~h}$; (j) $\mathrm{MeSH}, \mathrm{Py}$, benzene, $20-25^{\circ} \mathrm{C}, 5 \mathrm{~h} ; \mathrm{H}_{2} \mathrm{O}_{2}$ (excess), $\mathrm{AcOH}$, reflux, $4 \mathrm{~h}$; piperazine (excess), EtOH, reflux, $2 \mathrm{~h} ; \mathrm{HCl}$ (excess), $4{ }^{\circ} \mathrm{C}, 24 \mathrm{~h}$.

8 was active against leukemia HL-60(TB) $(\mathrm{GI}=49.92 \%$ and $\mathrm{K}-562(\mathrm{GP}=72.30 \%)$ as well as breast cancer MDAMB-468 (GP $=72.05 \%)$ cells. Compound 9 showed appreciable inhibitory activity only against lung cancer cells NCI-H522 (GI $=59.56 \%)$.

1,3-Thiazole 17 which bears a pyrazole ring instead of the benzene one at $\mathrm{C}^{2}$ showed lower inhibitory activity compared with that of the parent compound 1. It showed only minor activity towards breast cancer cells BT-549 ( $\mathrm{GP}=84.33 \%)$. On the other hand, the substitution of the chlorine atom in compound $\mathbf{1 7}$ with the propylsulfanyl group results in compound 20, which inhibitory activity towards lung cancer cells HOP-92 is significantly higher $(\mathrm{GP}=20.91 \%)$. The activity of compound $\mathbf{2 0}$ against the other cancer types remains at the level of compound $\mathbf{1 7 .}$ The replacement of the chlorine atom in compound $\mathbf{1 7}$ with the morpholine cycle does not increase the inhibitory activity. Compound $\mathbf{1 8}$ proved to be practically inactive towards all types of cancer cells.

Entering a furan cycle in position $\mathrm{C}^{2}$ of the 1,3-thiazole leads to a significant increase in the anticancer activity.
Thus, synthesized 5-chloro-2-(furan-2-yl)-4-tosyl-1,3thiazole (10) was active against leukemia (K-562, $\mathrm{GP}=18.83 \%$ and $\mathrm{SR}, \mathrm{GP}=8.28 \%)$, lung cancer $(\mathrm{NCI}-$ H522, GP $=18.49 \%$ ), melanoma (M14, GP $=32.33 \%$ and MDA-BM-435, GP $=4.90 \%$ ), and breast cancer (MDAMB-468, GP $=-22.87 \%$ ).

The most active were 1,3-thiazoles containing a piperazine ring in position $\mathrm{C}^{5}$. Thus, compound $\mathbf{1 9}$ was active against epithelial cancer cells HCC-2998 (GP = $-88.55 \%)$ and HT29 (GP $=-33.93 \%)$, almost all melanoma lines $($ MALME-3M, GP $=-55.98 \%$; M14, GP $=-89.83 \%$; MDA-MB-435, GP $=-2,08 \%$; SK-MEL-28, GP $=-83.71 \%$; SK-MEL-5, GP $=80.68 \%$; UACC-257, GP $=-79.19 \%$; UACC-62, GP $=72.05 \%$ ), and breast cancer as well $(\mathrm{T}-47 \mathrm{D}, \mathrm{GP}=-29.33 \%$; MDA-MB-468, GP $=-55.79 \%)$.

1,3-Thiazole 21 with two tosyl groups and $\mathrm{C}^{5}$ linked piperazine also showed high anticancer activity (average $\mathrm{GP}=-5.77 \%)$. The most effect was observed on leukemia HL-60 (TB) $(\mathrm{GP}=-45.35 \%)$, lung cancer NCI-H522 $(\mathrm{GP}=-44.16 \%)$ and $\mathrm{NCI}-\mathrm{H} 460(\mathrm{GP}=-38.88 \%)$, colon cancer COLO $205(\mathrm{GP}=-32.92 \%)$, CNS cancer SF-539 
$(\mathrm{GP}=-15.93 \%)$, melanoma LOX IMVI $(\mathrm{GP}=-41.57 \%)$, M14 (GP $=-49.08 \%)$ and MDA-MB-435 (GP $=-43.55 \%)$, prostate cancer DU-145 (GP $=-35.29 \%)$, breast cancer HS 578T $(\mathrm{GP}=-21.17 \%)$ and MDA-MB-468 (GP $=$ $-21.44 \%)$ cells.

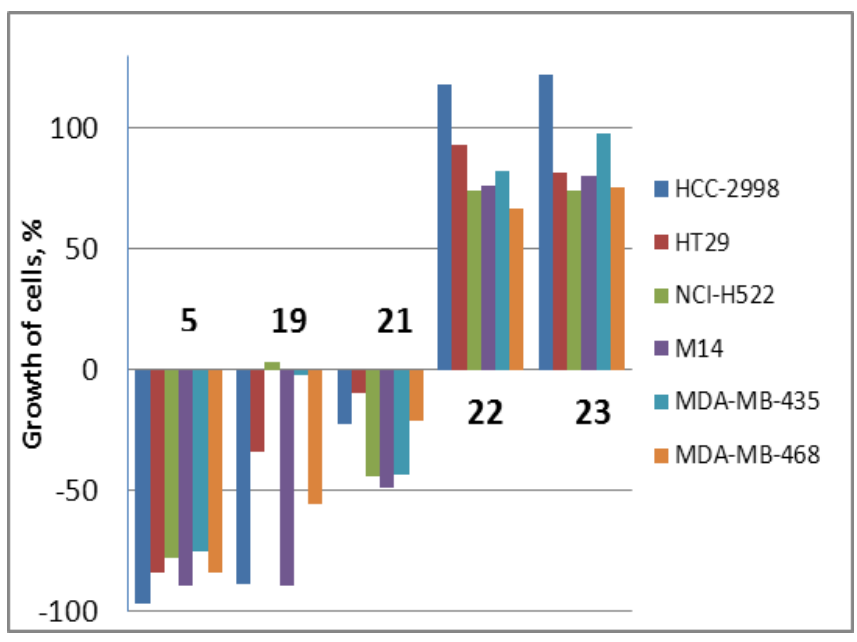

Figure 1. Antitumor activity of 5-(piperazin-1-yl)-4-tosyl-1,3thiazoles 5, 19, 21-23.

2-Phenyl-5-(piperazin-1-yl)-4-tosyl-1,3-thiazole (5) has been a prominent anticancer agent. It significantly decreased the growth of ovarian cancer cells (IGROV-1, $\mathrm{GP}=31.39 \%$ and OVCAR, GP $=49.75 \%$ ), and destroyed, with the average GP of $-70 \%$, almost all cell lines of leukemia, melanoma, and colon, CNS, kidney, and breast cancer. The most significant are the data reflecting the almost complete destruction of the following cell lines: colon cancer HCC2998 (GP = -96.68\%), CNS U251 $(\mathrm{GP}=-91.74 \%)$, melanoma SK-MEL-28 $(\mathrm{GP}=-97.03 \%)$ and SK-MEL-5 (GP = -98.77\%), kidney cancer TK-10 $(\mathrm{GP}=-88.46 \%)$, breast cancer MCF7 $(\mathrm{GP}=-84.29 \%)$ and MDA-MB-468 (GP = -84.34\%).

Advanced in vitro study of compounds 5, 19, 21 at five concentrations of the 10 -fold dilution $\left(10^{-4}-10^{-8} \mathrm{M}\right)$ was also performed towards 60 human cancer cell lines, the set of which was identical to that for the pre-screening stage (Table 2). High antitumor potential of compound $\mathbf{2 1}$ has been confirmed by a significant level of inhibition (average $\lg \mathrm{GI}_{50}=-5.67$ ), as well as cytostatic (average $1 \mathrm{~g}$ TGI = -5.21) and cytotoxic (average $\lg \mathrm{LC}_{50}=-4.67$ ) effects. The highest data were found for compound 5: average $\lg \mathrm{GI}_{50}=-5.87, \lg \mathrm{TGI}=-5.54$, and $\lg \mathrm{LC}_{50}=-5.21$.

It is of interest that among the 5-piperazino-substituted 1,3-thiazoles 5, 19, 21-23 compounds 22, 23 containing a $\mathrm{C}^{2}$ linked alkylsulfonyl group exhibit the lowest level of antitumor activity. This is readily illustrated by Figure 1 with some selected cell lines. The average activity value of compounds $\mathbf{2 2}$ and $\mathbf{2 3}$ was $95.48 \%$ and $98.61 \%$, respectively (Table 1).

\section{Conclusions}

The study of the antitumor activity of $\mathrm{di}^{-}$and trifunctionally substituted 1,3-thiazoles towards the NCI 60 human cancer cell lines revealed "leader compounds" 5-(piperazin-1-yl)-4-tosyl-1,3-thiazoles. Therein, the nature of the substituent at $\mathrm{C}^{2}$ of the 1,3-thiazole cycle critically affects the level of activity. Most preferred is the presence of phenyl, tosyl or 3,5-dimethyl-1H-pyrazol-1-yl substituent in this position.

Table 1. Mitotic activity of the 1,3-thiazole derivatives towards NCI 60 cell lines at the $10^{-5} \mathrm{M}$ concentration.

$\begin{array}{lll}\text { Compd } & \text { Structure } & \text { Average GP }\end{array}$

1<smiles>Clc1sc(-c2ccccc2)nc1[Hg]</smiles>

97.48

100.29 leukemia K-562 (30.07), SR (13.60);

lung cancer $\mathrm{NCI}-\mathrm{H} 522$ (44.91); melanoma M-14 (37.06), MDA-MB-435 (1.37); breast cancer MDA-MB-468 (0.73)

leukemia K-562 (90.20), SR (88.33); lung cancer $\mathrm{NCI}-\mathrm{H} 522$ (83.67); melanoma M-14 (109.28), MDA-MB-435 (119.03); kidney cancer CAKI-1 (71.89)

leukemia K-562 (103.76), SR (88.33); lung cancer $\mathrm{NCI}-\mathrm{H} 522$ (75.91); melanoma MDA-MB-435 (106.38); breast cancer T-47D (70.39)

colon cancer HCC-2998 (-96.68); melanoma SK-MEL-5 (-98.77); CNS cancer U251 (-91.74); breast cancer MDA-MB-468 (-84.34) 
Table 1. (Contd.)

$\begin{array}{llll}\text { Compd } & \text { Structure } & \text { Average GP } & \text { The most sensitive cell lines (GP) }\end{array}$

6<smiles>CSc1sc(-c2ccccc2)nc1[Hg]</smiles>

7<smiles>[3H]c1nc(-c2ccccc2)sc1Oc1ccccc1</smiles>

8<smiles>Oc1sc(-c2ccccc2)nc1N1CCOCC1</smiles>

9

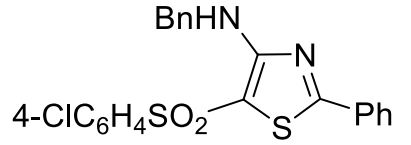

10<smiles>Sc1nc(-c2ccco2)sc1Cl</smiles>

11<smiles>[3H]c1nc(C)sc1OS(=O)(=O)OC(C)(Cl)Cl</smiles>

12<smiles>[3H]c1nc(NCc2ccccc2)sc1OS(=O)(=O)OC(C)(C)C</smiles>

13<smiles>CC(Cl)(Cl)OS(=O)(=O)Oc1sc(N)nc1[As]</smiles>

14<smiles>CCCCOc1sc(N2CCCCC2)nc1N1CCCCC1</smiles>

BnHN

15

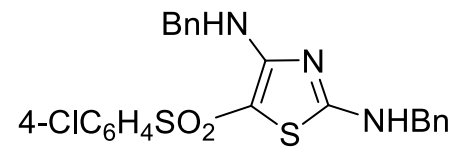

leukemia K-562 (58.49), SR (49.84); lung cancer NCI-H522 (74.11);

melanoma M-14 (94.70), MDA-MB-435 (36.78); breast cancer MDA-MB-468 (65.56)

leukemia SR (95.44);

lung cancer NCI-H522 (85.13); melanoma M-14 (101.29), MDA-MB-435 (107.97); breast cancer T-47D (54.11)

leukemia HL-60(TB) (49.92), SR (92.70);

lung cancer NCI-H522 (83.12); melanoma M-14 (101.29), MDA-MB-435 (107.63); breast cancer MDA-MB-468 (72.05)

leukemia K-562 (88.04), SR (77.65);

lung cancer NCI-H522 (59.56); melanoma M-14 (100.66), MDA-MB-435 (107.82); breast cancer MDA-MB-468 (96.90)

leukemia K-562 (18.83), SR (8.28); lung cancer NCI-H522 (18.49); melanoma M-14 (32.33), MDA-MB-435 (4.90); breast cancer MDA-MB-468 (-22.87)

leukemia HL-60(TB) (52.69); lung cancer A549/ATCC (56.18); melanoma UACC62 (59.80); breast cancer MDA-MB-468 (66.25)

CNS cancer SNB-75 (64.32); melanoma UACC-62 (79.75)

leukemia CCRF-CEM (72.45), HL-60(TB) (72.65), K-562 (73.24), MOLT (64.64); lung cancer HOP-92 (47.03)

leukemia K-562 (114.19), SR (100.78); lung cancer NCI-H522 (91.60); melanoma M-14 (106.27); breast cancer MDA-MB-468 (94.07); CNS cancer $\mathrm{SNB}-75$ (73.04) melanoma M-14 (77.99), MDA-MB-435 (114.47); breast cancer MDA-MB-231/ATCC (42.35) 
Table 1. (Contd.)

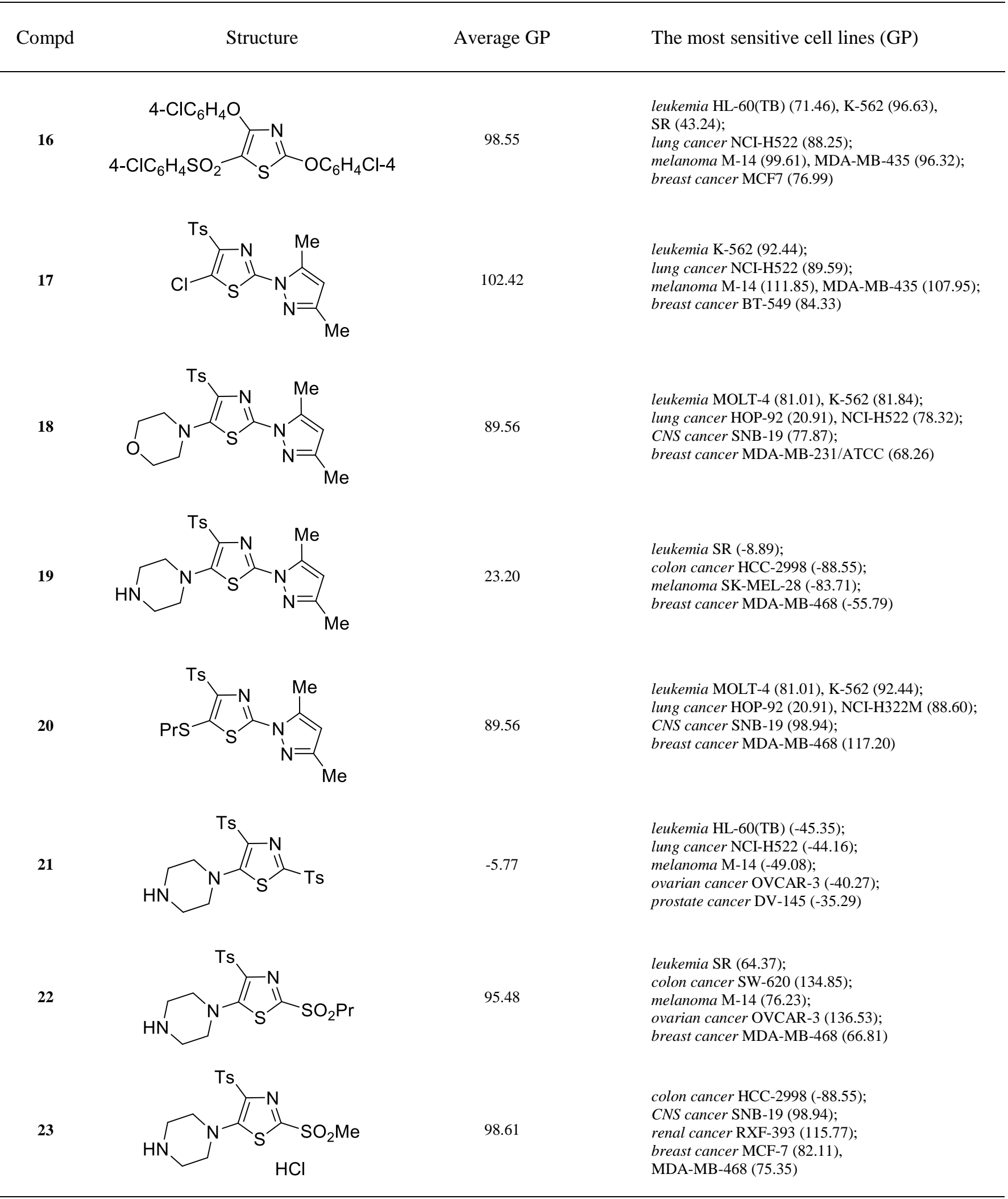


Table 2. Parameter values ( $\mathrm{lg}$ ) of the anticancer activity of compounds 5, 19, 21 against the NCI 60 human cancer cell lines (five-dose assay).

\begin{tabular}{|c|c|c|c|c|c|c|c|c|c|}
\hline \multirow{3}{*}{ Cell Line } & \multicolumn{9}{|c|}{ Compd } \\
\hline & \multicolumn{3}{|c|}{5} & \multicolumn{3}{|c|}{19} & \multicolumn{3}{|c|}{21} \\
\hline & $\mathrm{GI}_{50}$ & TGI & $\mathrm{LC}_{50}$ & $\mathrm{GI}_{50}$ & TGI & $\mathrm{LC}_{50}$ & $\mathrm{GI}_{50}$ & TGI & $\mathrm{LC}_{50}$ \\
\hline & \multicolumn{9}{|c|}{ Leukemia } \\
\hline CCRF-CEM & -5.73 & -5.28 & -4.23 & -5.57 & -4.96 & -4.21 & -5.63 & -5.05 & -4.35 \\
\hline HL-60(TB) & -5.72 & -5.43 & -5.14 & -5.62 & -5.35 & -5.07 & -5.64 & -5.32 & -5.00 \\
\hline K-562 & -6.26 & -5.62 & -5.11 & -5.81 & -5.39 & -4.91 & NT & NT & NT \\
\hline MOLT-4 & -5.74 & -5.39 & -5.03 & -5.65 & -5.31 & -4.88 & -5.52 & -5.23 & -4.53 \\
\hline RPMI-8226 & NT & NT & NT & NT & NT & NT & -5.94 & -5.01 & -4.19 \\
\hline \multirow[t]{2}{*}{ SR } & -5.87 & -5.47 & -5.08 & -5.64 & -5.24 & -4.49 & -5.63 & -5.31 & -4.38 \\
\hline & \multicolumn{9}{|c|}{ Non-small cell lung cancer } \\
\hline A549/ATCC & -5.74 & -5.47 & -5.20 & -5.44 & -4.89 & -4.38 & -5.64 & -5.10 & -4.48 \\
\hline EKVX & -5.87 & -5.52 & -5.16 & -5.63 & -4.90 & -4.39 & -5.49 & -4.91 & -4.40 \\
\hline HOP-62 & -5.77 & -5.51 & -5.26 & -5.71 & -5.39 & -5.07 & -5.92 & -5.39 & -4.70 \\
\hline HOP-92 & -6.46 & -5.79 & -5.37 & -6.21 & -5.50 & -4.83 & -5.45 & -5.12 & -4.57 \\
\hline NCI-H226 & -5.79 & -5.51 & -5.23 & -5.57 & -5.00 & -4.38 & -5.67 & -5.37 & -5.06 \\
\hline NCI-H23 & -5.84 & -5.53 & -5.23 & -5.29 & -4.71 & -4.34 & -5.63 & -5.17 & -4.55 \\
\hline NCI-H322M & NT & NT & NT & NT & NT & NT & NT & NT & NT \\
\hline NCI-H460 & -5.79 & -5.52 & -5.24 & -5.74 & -5.45 & -5.16 & -5.64 & -5.20 & -4.55 \\
\hline \multirow[t]{2}{*}{ NCI-H522 } & -5.74 & -5.46 & -5.18 & -5.67 & -5.39 & -5.10 & NT & NT & NT \\
\hline & \multicolumn{9}{|c|}{ Colon cancer } \\
\hline COLO 205 & -6.68 & -6.33 & -5.95 & -5.78 & -5.52 & -5.25 & -5.78 & -5.45 & -5.12 \\
\hline HCC-2998 & -6.28 & 5.76 & -5.34 & -5.81 & -5.52 & -5.22 & -5.59 & 5.22 & -4.66 \\
\hline HCT-116 & -5.93 & -5.61 & -5.29 & -5.79 & -5.49 & -5.18 & -5.75 & -5.39 & -5.04 \\
\hline НCT-15 & -5.84 & -5.54 & -5.24 & -5.67 & -5.31 & -4.86 & -5.70 & -5.07 & -4.47 \\
\hline HT29 & -6.12 & -5.65 & -5.24 & -5.82 & -5.40 & -4.95 & -5.56 & -4.98 & -4.30 \\
\hline KM12 & -5.79 & -5.51 & -5.24 & -5.74 & -5.48 & -5.22 & -5.61 & -5.18 & -4.50 \\
\hline \multirow[t]{2}{*}{ SW-620 } & -5.82 & -5.54 & -5.26 & -5.77 & -5.48 & -5.19 & -5.79 & -5.44 & -5.09 \\
\hline & \multicolumn{9}{|c|}{ CNS cancer } \\
\hline SF-268 & -5.80 & -5.50 & -5.20 & -5.48 & -4.92 & -4.43 & -5.58 & -5.09 & -4.46 \\
\hline SF-295 & -5.77 & -5.49 & -5.21 & -5.73 & -5.43 & -5.14 & -5.60 & -5.19 & -4.59 \\
\hline SF-539 & -5.81 & -5.53 & -5.24 & -5.77 & -5.46 & -5.14 & -5.56 & -5.25 & -4.83 \\
\hline SNB-19 & -5.75 & -5.36 & -4.88 & -5.16 & -4.68 & -4.30 & -5.51 & -4.95 & -4.45 \\
\hline SNB-75 & -5.97 & -5.62 & -5.27 & -5.85 & -5.39 & -4.87 & -5.59 & -5.09 & -4.54 \\
\hline \multirow[t]{2}{*}{$\mathrm{U} 251$} & NT & NT & NT & NT & NT & NT & -5.55 & -5.01 & -4.45 \\
\hline & \multicolumn{9}{|c|}{ Melanoma } \\
\hline LOX IMVI & -5.88 & -5.57 & -5.26 & -5.75 & -5.45 & -5.15 & -5.82 & -5.39 & -4.86 \\
\hline MALME-3M & -5.77 & -5.50 & -5.22 & -5.33 & -4.79 & -4.36 & -5.61 & -5.34 & -5.06 \\
\hline M14 & -5.76 & -5.47 & -5.19 & -5.67 & -5.41 & -5.16 & -5.80 & -5.48 & -5.15 \\
\hline MDA-MB-435 & -5.87 & -5.55 & -5.24 & -5.75 & -5.45 & -5.16 & -6.23 & -5.65 & -5.17 \\
\hline SK-MEL-2 & -5.69 & -5.42 & -5.15 & -5.65 & -5.40 & -5.15 & NT & NT & NT \\
\hline SK-MEL-28 & -5.81 & -5.54 & -5.27 & -5.76 & -5.50 & -5.24 & -5.74 & -5.44 & -5.14 \\
\hline SK-MEL-5 & -5.82 & -5.55 & -5.27 & -5.78 & -5.52 & -5.26 & -5.84 & -5.55 & -5.27 \\
\hline UACC-257 & -5.80 & -5.50 & -5.19 & -5.77 & -5.48 & -5.20 & -5.79 & -5.48 & -5.17 \\
\hline \multirow[t]{2}{*}{ UACC-62 } & -5.80 & -5.53 & -5.26 & -5.77 & -5.49 & -5.22 & -5.79 & -5.28 & -4.68 \\
\hline & \multicolumn{9}{|c|}{ Ovarian cancer } \\
\hline IGROV1 & -5.75 & -5.44 & -5.14 & -5.74 & -5.45 & -5.17 & -5.60 & -5.13 & -4.37 \\
\hline OVCAR-3 & -5.77 & -5.50 & -5.23 & -5.59 & -5.15 & -4.59 & -5.61 & -5.28 & -4.83 \\
\hline OVCAR-4 & -5.76 & -5.46 & -5.16 & -5.38 & -4.81 & -4.40 & -5.56 & -4.92 & -4.39 \\
\hline OVCAR-5 & -5.80 & -5.51 & -5.22 & -5.50 & -4.95 & -4.46 & -5.44 & -5.05 & -4.51 \\
\hline OVCAR-8 & -5.75 & -5.46 & -5.17 & -5.64 & -5.22 & -4.47 & -5.65 & -4.99 & -4.35 \\
\hline NCI/ADR-RES & -5.81 & -5.49 & -5.17 & -5.50 & -4.84 & -4.32 & -5.55 & -4.92 & -4.34 \\
\hline SK-OV-3 & -5.76 & -5.49 & -5.23 & -5.53 & -5.11 & -4.57 & -5.50 & -4.97 & -4.43 \\
\hline
\end{tabular}


Table 2. (Contd.)

\section{Compd}

\begin{tabular}{|c|c|c|c|c|c|c|c|c|c|}
\hline \multirow{3}{*}{ Cell Line } & \multirow{2}{*}{\multicolumn{3}{|c|}{5}} & \multirow{2}{*}{\multicolumn{3}{|c|}{19}} & \multirow{2}{*}{\multicolumn{3}{|c|}{21}} \\
\hline & & & & & & & & & \\
\hline & $\mathrm{GI}_{50}$ & TGI & $\mathrm{LC}_{50}$ & $\mathrm{GI}_{50}$ & TGI & $\mathrm{LC}_{50}$ & $\mathrm{GI}_{50}$ & TGI & $\mathrm{LC}_{50}$ \\
\hline & \multicolumn{9}{|c|}{ Renal cancer } \\
\hline $786-0$ & -5.79 & -5.48 & -5.17 & -5.66 & -5.29 & -4.77 & -5.65 & -5.37 & -5.08 \\
\hline A498 & -5.00 & -5.66 & -5.33 & -5.91 & -5.50 & -5.08 & -5.74 & -5.34 & -4.84 \\
\hline $\mathrm{ACHN}$ & -5.78 & -5.50 & -5.21 & -5.46 & -4.92 & -4.45 & -5.41 & -4.91 & -4.44 \\
\hline CAKI-1 & -5.82 & -5.50 & -5.18 & -5.68 & -5.23 & -4.65 & -5.66 & -5.34 & -5.03 \\
\hline RXF 393 & -5.90 & -5.59 & -5.29 & -5.77 & -5.45 & -5.12 & -5.80 & -5.52 & -5.24 \\
\hline SN12C & -5.80 & -5.51 & -5.23 & -5.63 & -5.15 & -4.54 & -5.57 & -4.99 & -4.47 \\
\hline TK-10 & -5.76 & -5.48 & -5.20 & -5.58 & -5.19 & -4.66 & -5.42 & -4.99 & -4.46 \\
\hline \multirow[t]{2}{*}{ UO-31 } & -5.94 & -5.61 & -5.29 & -5.88 & -5.54 & -5.20 & -5.49 & -5.00 & -4.45 \\
\hline & \multicolumn{9}{|c|}{ Prostate cancer } \\
\hline PC-3 & -5.81 & -5.48 & -5.15 & -5.52 & -4.99 & -4.46 & -5.51 & -4.88 & -4.41 \\
\hline \multirow[t]{2}{*}{ DU-145 } & -5.79 & -5.52 & -5.25 & -5.43 & -4.88 & -4.44 & -5.66 & -5.28 & -4.76 \\
\hline & \multicolumn{9}{|c|}{ Breast cancer } \\
\hline MCF7 & -5.84 & -5.55 & -5.26 & -5.76 & -5.43 & -5.10 & -5.78 & -5.21 & -4.44 \\
\hline MDA-MB-231/ATCC & -5.83 & -5.54 & -5.25 & -5.74 & -5.44 & -5.13 & -5.54 & -5.16 & -4.62 \\
\hline HS 578T & -5.81 & -5.45 & -5.09 & -5.68 & -5.24 & -4.25 & -5.61 & -5.14 & -4.00 \\
\hline BT-549 & -5.78 & 5.51 & 5.23 & -5.47 & -4.91 & -4.43 & -5.52 & 5.11 & 4.57 \\
\hline $\mathrm{T}-47 \mathrm{D}$ & -5.80 & -5.50 & -5.19 & -5.76 & -5.46 & -5.16 & -6.29 & -5.18 & -4.43 \\
\hline MDA-MB-468 & -6.56 & -6.01 & -5.44 & -5.92 & -5.61 & -5.30 & -6.43 & -5.77 & -5.23 \\
\hline
\end{tabular}

\section{Experimental section}

\section{Chemistry}

${ }^{1} \mathrm{H}$ NMR spectra were obtained on a Bruker Avance DRX 500 spectrometer. The melting points were estimated on a Fisher-Johns apparatus. The reaction progress was monitored by the TLC method on silica gel $60 \mathrm{~F}_{254}$ Merck plates. All reagents and solvents were purchased from Aldrich and used without additional purification.

5-Substituted 4-[(4-methylphenyl)sulfonyl]-2-phenyl-1,3thiazoles 1-4, 6-9 [11], 5-Chloro-2-(furan-2-yl)-4-((4methylphenyl)sulfonyl)-1,3-thiazole (10) [12], 2,4-Disubstituted 5-(4-chlorophenyl)sulfonyl-1,3-thiazoles 11-16 [13], 2-(3,5-Dimethyl-1H-pyrazol-1-yl)-4-((4-methylphenyl)sulfonyl)-1,3-thiazoles 17, 18 [14] were synthesized following the procedures described in the corresponding sources cited.

\section{1-(4-((4-Methylphenyl)sulfonyl)-2-phenyl-1,3-thiazol-5-} yl)piperazine (5) was synthesized similarly to compound 4.

Yield $67 \%$, mp $112-113{ }^{\circ} \mathrm{C}(\mathrm{EtOH}) .{ }^{1} \mathrm{H}$ NMR $(500 \mathrm{MHz}$, $\left.\mathrm{CDCl}_{3}\right) \delta 2.45\left(\mathrm{~s}, 3 \mathrm{H}, \mathrm{CH}_{3}\right), 3.20-3.35\left(\mathrm{~m}, 4 \mathrm{H}, 2 \mathrm{CH}_{2}\right), 3.82-$ $3.94\left(\mathrm{~m}, 4 \mathrm{H}, 2 \mathrm{CH}_{2}\right), 7.33-7.45(\mathrm{~m}, 5 \mathrm{H}, \mathrm{Ar}), 7.78(\mathrm{~d}, J 7.5$ $\mathrm{Hz}, 2 \mathrm{H}, \mathrm{Ar}), 8.05$ (d, J 7.5 Hz, 2H, Ar). Anal. Calcd. for $\mathrm{C}_{20} \mathrm{H}_{21} \mathrm{~N}_{3} \mathrm{O}_{2} \mathrm{~S}_{2}$ : C, 60.12; H, 5.30; N, 10.52; S, 16.05 . Found: C, 59.98; H, 5.15; N, 10.32; S, 15.98 .

\section{1-(2-(3,5-Dimethyl-1H-pyrazol-1-yl)-4-((4-methylphe-} nyl)sulfonyl)-1,3-thiazol-5-yl)piperazine (19) was synthesized similarly to compound $\mathbf{1 8}$.
Yield 62\%, mp 98-99 ${ }^{\circ} \mathrm{C}(\mathrm{EtOH}) .{ }^{1} \mathrm{H}$ NMR $(500 \mathrm{MHz}$, $\left.\mathrm{CDCl}_{3}\right) \delta 2.17\left(\mathrm{~s}, 3 \mathrm{H}, \mathrm{CH}_{3}\right), 2.40\left(\mathrm{~s}, 3 \mathrm{H}, \mathrm{CH}_{3}\right), 2.44(\mathrm{~s}, 3 \mathrm{H}$, $\left.\mathrm{CH}_{3}\right)$, 3.79-3.88 (m, 4H, 2CH$), 4.12-4.23\left(\mathrm{~m}, 4 \mathrm{H}, 2 \mathrm{CH}_{2}\right)$, 6.00 (s, 1H, CH), 7.38 (d, J $7.9 \mathrm{~Hz}, 2 \mathrm{H}, \mathrm{Ar}), 7.82$ (d, J 7.9 $\mathrm{Hz}, 2 \mathrm{H}$, Ar). Anal. Calcd. for $\mathrm{C}_{19} \mathrm{H}_{23} \mathrm{~N}_{5} \mathrm{O}_{2} \mathrm{~S}_{2}$ : C, 54.65; H, 5.55; N, 16.77; S, 15.36. Found: C, 54.48; H, 5.49; N, 16.54; S, 15.12 .

\section{2-(3,5-Dimethyl-1H-pyrazol-1-yl)-4-((4-methylphenyl)-} sulfonyl)-5-(propylsulfanyl)-1,3-thiazole (20).

To a suspension of $0.00015 \mathrm{~mol}$ of compound $\mathbf{1 7}$ in 10 $\mathrm{ml}$ of methanol, $0.00075 \mathrm{~mol}$ of sodium hydrosulfide was added. The mixture was stirred for $20 \mathrm{~h}$ at $20^{\circ} \mathrm{C}$, the precipitate was filtered off, and the filtrate was evaporated in vacuo. To the residue, $5 \mathrm{ml}$ of water followed by $1 \mathrm{ml}$ of concd hydrochloric acid was added to precipitate a solid, which was filtered off. To a suspension of this solid in $5 \mathrm{ml}$ of methanol, $0.00015 \mathrm{~mol}$ of sodium methylate followed by $0.0002 \mathrm{~mol}$ of propyl bromide was added. The mixture was refluxed for $3 \mathrm{~h}$ then cooled to $20{ }^{\circ} \mathrm{C}$, the precipitate was filtered off and recrystallized from ethanol. Yield 65\%, mp 142-144 ${ }^{\circ} \mathrm{C}(\mathrm{EtOH})$. Anal. Calcd. for $\mathrm{C}_{18} \mathrm{H}_{21} \mathrm{~N}_{3} \mathrm{O}_{2} \mathrm{~S}_{3}$ : C, 53.04; H, 5.19; N, 10.31; S, 23.60. Found: C, 52.91; H, 5.00; N, 10.18; S, 23.54.

General procedure for preparation of compounds (21, 22).

To a solution of $0.08 \mathrm{~mol}$ of compound II [15] in $150 \mathrm{ml}$ of benzene cooled to $0{ }^{\circ} \mathrm{C}, 0.08 \mathrm{~mol}$ of 4-methylbenzenethiol or propanethiol followed by $0.08 \mathrm{~mol}$ of pyridine was added. The mixture was stirred for $8 \mathrm{~h}$ at $15^{\circ} \mathrm{C}$, the solvent was removed in vacuo, the residue was 
washed with water, and dissolved in $200 \mathrm{ml}$ of acetic acid. To this solution, $30 \mathrm{ml}$ of a $30 \%$ aqueous hydrogen peroxide solution was added, the reaction mixture was boiled for $4 \mathrm{~h}$ then cooled to $10^{\circ} \mathrm{C}$. The precipitate was filtered off and dissolved in $50 \mathrm{ml}$ of ethanol. To this solution, $0.25 \mathrm{~mol}$ of piperazine was added, the reaction mixture was boiled for $1 \mathrm{~h}$ then evaporated in vacuo. To the residue, $20 \mathrm{ml}$ of water was added to precipitate a solid, which was filtered off and recrystallized from ethanol.

\section{1-(2,4-Bis((4-methylphenyl)sulfonyl)-1,3-thiazol-5-yl)- piperazine (21).}

Yield $72 \%$, mp $132-133{ }^{\circ} \mathrm{C}(\mathrm{EtOH}) .{ }^{1} \mathrm{H}$ NMR $(500 \mathrm{MHz}$, $\left.\mathrm{CDCl}_{3}\right) \delta 2.43\left(\mathrm{~s}, 3 \mathrm{H}, \mathrm{CH}_{3}\right), 2.47\left(\mathrm{~s}, 3 \mathrm{H}, \mathrm{CH}_{3}\right), 3.80-3.89$ $\left(\mathrm{m}, 4 \mathrm{H}, 2 \mathrm{CH}_{2}\right), 4.15-4.30\left(\mathrm{~m}, 4 \mathrm{H}, 2 \mathrm{CH}_{2}\right), 7.29(\mathrm{~d}, J 7.6 \mathrm{~Hz}$, 2H, Ar), 7.35 (d, J 7.9 Hz, 2H, Ar), 7.38 (d, J 7.6 Hz, 2H, Ar), 7.86 (d, J $7.9 \mathrm{~Hz}, 2 \mathrm{H}$, Ar). Anal. Calcd. for $\mathrm{C}_{21} \mathrm{H}_{23} \mathrm{~N}_{3} \mathrm{O}_{4} \mathrm{~S}_{3}$ : C, 52.70; H, 4.78; N, 8.80; S, 20.14. Found: C, 52.81; H, 4.85; N, 8.63; S, 20.04.

1-(4-((4-Methylphenyl)sulfonyl)-2-(propylsulfonyl)-1,3thiazol-5-yl)piperazine (22).

Yield 68\%, mp 131-132 ${ }^{\circ} \mathrm{C}(\mathrm{EtOH}) .{ }^{1} \mathrm{H}$ NMR $(500 \mathrm{MHz}$, $\left.\mathrm{CDCl}_{3}\right) \delta 0.91\left(\mathrm{t}, J 7.4 \mathrm{~Hz}, 3 \mathrm{H}, \mathrm{CH}_{3}\right), 1.55-1.68(\mathrm{~m}, 2 \mathrm{H}$, $\mathrm{CH}_{2}$ ), 2.45 (s, 3H, $\mathrm{CH}_{3}$ ), 3.02-3.18 (m, 4H, 2CH$), 3.19-$ $3.31\left(\mathrm{~m}, 4 \mathrm{H}, 2 \mathrm{CH}_{2}\right), 3.33-3.45\left(\mathrm{~m}, 4 \mathrm{H}, 2 \mathrm{CH}_{2}\right), 7.34(\mathrm{~d}, J 7.5$ $\mathrm{Hz}, 2 \mathrm{H}, \mathrm{Ar}$ ), 7.89 (d, J 7.5 Hz, 2H, Ar). Anal. Calcd. for $\mathrm{C}_{17} \mathrm{H}_{23} \mathrm{~N}_{3} \mathrm{O}_{4} \mathrm{~S}_{3}$ : C, 47.53; H, 5.03; N, 9.78; S, 22.39. Found: C, 47.45; H, 4.93; N, 9.65; S, 22.10.

\section{1-(4-((4-Methylphenyl)sulfonyl)-2-(methylsulfonyl)-1,3- thiazol-5-yl)piperazine (23).}

To a solution of $0.0026 \mathrm{~mol}$ of isothiocyanate II [15] and $00078 \mathrm{~mol}$ of pyridine in $10 \mathrm{ml}$ of benzene, methanethiol was passed, obtained by the hydrolysis of $0.012 \mathrm{~mol}$ of $S$-methyl-isothiouronium sulfate. The mixture was stirred for $5 \mathrm{~h}$, the precipitate was filtered off, and the filtrate was evaporated in vacuo. To the residue, $4 \mathrm{ml}$ of acetic acid followed by $1.5 \mathrm{ml}$ of $30 \%$ aqueous hydrogen peroxide solution was added. The reaction mixture was heated under reflux for $1.5 \mathrm{~h}$ then cooled to room temperature and the precipitate was separated. To a suspension of this solid in $5 \mathrm{ml}$ of acetonitrile, $0.005 \mathrm{~mol}$ of piperazine was added. The mixture was stirred for $20 \mathrm{~h}$ and the precipitate was filtered off. To the filtrate, $0.5 \mathrm{ml}$ of concd hydrochloric acid was added, the mixture was kept at $4{ }^{\circ} \mathrm{C}$ for 1 day, and the precipitate was separated. Yield 25\%, mp $245-247{ }^{\circ} \mathrm{C}$ (dec.). ${ }^{1} \mathrm{H}$ NMR (500 MHz, DMSO-d 6 ) $\delta 2.39\left(\mathrm{~s}, 3 \mathrm{H}, \mathrm{CH}_{3}\right), 3.28\left(\mathrm{~s}, 4 \mathrm{H}, 2 \mathrm{CH}_{2}\right), 3.36\left(\mathrm{~s}, 3 \mathrm{H}, \mathrm{CH}_{3}\right)$, $3.56\left(\mathrm{~s}, 4 \mathrm{H}, 2 \mathrm{CH}_{2}\right), 7.46(\mathrm{~d}, J 8.0 \mathrm{~Hz}, 2 \mathrm{H}, \mathrm{Ar}), 7.86(\mathrm{~d}, J 8.1$ $\mathrm{Hz}, 2 \mathrm{H}, \mathrm{Ar}), 9.54(\mathrm{~s}, 2 \mathrm{H}, \mathrm{NH}, \mathrm{HCl})$. Anal. Calcd. for $\mathrm{C}_{15} \mathrm{H}_{20} \mathrm{ClN}_{3} \mathrm{O}_{4} \mathrm{~S}_{3}: \mathrm{C}, 41.13 ; \mathrm{H}, 4.60 ; \mathrm{Cl}, 8.09 ; \mathrm{N}, 9.59$; S, 21.96. Found: C, 41.24; H, 4.57; Cl, 8.17; N, 9.65; S, 21.85 .

\section{Biological tests}

Anticancer in vitro screening methodology as well as data interpretation rules is described in details at the NCI Development Therapeutics Program site [16].

\section{Notes}

Acknowledgements. We would like to thank National Cancer Institute, Bethesda, MD, US for the anticancer activity investigations and Enamine Ltd. for the material and technical support.

\section{References}

1. Forlani, L.; Todesco, P. E. In Thiazole and Its Derivatives, Part 1, Metzger, J. V., Ed.; The Chemistry of Heterocyclic Compounds, Weissberger, A.; Taylor, E. C., Eds.; Wiley: New York, USA, 1979; Vol. 34, pp 567-571.

2. Medicines, Mashkovsky, M. D., Ed., New Wave: Moscow, 2005.

3. Ji, K.; Choi, K.; Lee, S.; Park, S.; Khim, J. S.; Jo, E. H.; Choi, K.; Zhang, X.; Giesy, J. P. Effects of sulfathiazole, oxytetracycline and chlortetracycline on steroidogenesis in the human adrenocarcinoma (H295R) cell line and freshwater fish Oryzias latipes. J. Hazardous Materials 2010, 182, 494-502.

4. Felise, H. B.; Nguyen, H. V.; Pfuetzner, R. A.; Barry, K. C.; Jackson, S. R.; Blanc, M. P.; Bronstein, Ph. A.; Kline, T.; Miller, S. I. An Inhibitor of Gram-Negative Bacterial Virulence Protein Secretion. Cell Host \& Microbe 2008, 4, 325-336.

5. Kovalishyn, V.; Grouleff, J.; Semenyuta, I.; Sinenko, V.; Slivchuk, S.; Hodyna, D.; Brovarets, V.; Blagodatny, V.; Poda, G.; Tetko, I.; Metelytsia, L. Rational design of isonicotinic acid hydrazide derivatives with antitubercular activity: Machine learning, molecular docking, synthesis and biological testing. Chem. Biol. Drug Des. 2018, 92, 1272-1278.

6. Mak, J. Y. W.; Xu, W.; Fairlie, D. P. In Peptidomimetics I, Lubell, W. D., Ed.; Springer, 2015; pp 235-266.

7. Alley, M. C.; Scudiero, D. S.; Monks, P. A.; Hursey, M. L.; Czerwinski, M. J.; Fine, D. L.; Abbott, B. J.; Mayo, J. G.; Shoemaker, R. H.; Boyd, M. R. Feasibility of Drug Screening with Panels of Human Tumor Cell Lines Using a Microculture Tetrazolium Assay. Cancer Research 1988, 48, 589-601.

8. Grever, M. R.; Schepartz, S. A.; Chabner, B. A. The National Cancer Institute: cancer drug discovery and development program. Seminars in Oncology 1992, 19, 622-638.

9. Boyd, M. R.; Paull, K. D. Some practical considerations and applications of the national cancer institute in vitro anticancer drug discovery screen. Drug Development Research 1995, 34, 91-109.

10. Shoemaker, R. H. The NCI60 human tumour cell line anticancer drug screen. Nature Reviews 2006, 6, 813-823.

11. Turov, K. V.; Vinogradova, T. K.; Brovarets, V. S.; Drach, B. S. Reactions of 4-tosyl-2-phenyl-5-chloro-1, 3-thiazole with $N$-, $O$-, and S-nucleophiles. Russ. J. Gen. Chem. 2010, 80, 825-828.

12. Turov, K. V.; Vinogradova, T. K.; Rusanov, E. B. et al. Reaction of 1-tosyl-2,2-dichloroenamines with the Lawesson's reagent. Russ. J. Gen. Chem. 2012, 82, 848-852.

13. Turov, K. V.; Drach. B. S. Reaction of 1-tosyl-2, 2-dichloroenamines with the Lawesson's reagent. Russ. J. Gen. Chem. 2008, 78, 629-633.

14. Turov, K. V.; Vinogradova, T. K.; Drach, B. S. Transformations of 5-chloro-2-hydrazino-4-p-tolylsulfonyl-1,3-thiazole. Russ. J. Gen. Chem. 2008, 78, 2132-2136.

15. Babii, S. B.; Zyabrev, V. S.; Drach, B. S. Cyclocondensation of 1Tosyl-2,2-dichloroethenyl Isothiocyanate with $\mathrm{O}-, \mathrm{S}-, \mathrm{N}$-, and $\mathrm{C}$ Nucleophiles. Russ. J. Gen. Chem. 2002, 72, 1730-1735.

16. NCI-60 Human Tumor Cell Lines Screen. DTP Developmental Therapeutics Program, NIH website [Internet]. Available from: https://dtp.cancer.gov/discovery development/nci-60/default.htm (accessed on March 20, 2020). 


\title{
Оцінка протиракової активності ди- та трифункціональнозаміщених 1,3-тіазолів
}

\author{
К. В. Туров, О. П. Мітюхін, С. А. Чумаченко, В. С. Зябрєв, В. С. Броварець*
}

Інститут біоорганічної хімії та нафтохімї ім. В.П. Кухаря НАН України, вул. Мурманська, 1, Київ, О2094, Украӥна.

Резюме: Синтезовано ряд ди- та трифункціональнозаміщених 1,3-тіазолів з використанням в якості вихідних сполук 1-R-3-тозил-1,4,4-трихлоро2-аза-1,3-бутадієнів або 1-тозил-2,2-дихлороетенілізотіоціанату. Скринінгові дослідження протиракової активності синтезованих сполук проведено in vitro на 60 лініях ракових клітин людини: лейкемії (лінії CCRF-CEM, HL-60 (TB), K-562, MOLT-4, RPMI-8226, SR), меланоми (лінії LOX IMVI, MALME-3M, M14, MDA-MB-435, SK-MEL-2, SK-MEL-28, SK-MEL-5 , UACC-257, UACC-62), раку легенів (лінії A549/ATCC, ЕKVX, HOP-62, HOP-92, NCI-H226, NCI-H23, NCI-H322M, NCI-H460, NCI-H522), товстої кишки (лінії COLO 205, HCC-2998, HCT-116, HCT-15, HT29, KM12, SW-620), мозку (лінії SF-268, SF-295, SF-539, SNB-19, SNB-75, U251), яєчників (лінії IGROV1, OVCAR-3, OVCAR-4, OVCAR-5, OVCAR8, NCI/ADR-RES, SK-OV-3), нирок (лінії 786-0, A498, ACHN, CAKI-1, RXF 393, SN12C, TK-10, UO-31), простати (лінії PC-3, DU-145) і грудей (лінії MCF7, MDA-MB-231/ATCC, HS 578T, ВT-549, T- 47D, MDA-MB-468) при концентрації $1 \cdot 10^{-5}$ М. В результаті визначено відсоток росту (GP) клітин ліній раку у порівнянні з контролем (контроль - 100\%). Поглиблений in vitro скринінг сполук полягав у вивченні ії протипухлинного ефекту в п`яти концентраціях при 10 -кратному розведенні $\left(10^{-4}-10^{-8} \mathrm{M}\right)$. У результаті експерименту розраховано 3 дозозалежні параметри (GI ${ }_{50}$, TGI, LC 50 . Серед даних сполук 1-(4-((4-метилфеніл)сульфоніл)-2-феніл-1,3-тіазол-5-іл)піперазин (середні значення $1 \mathrm{~g} \mathrm{GI} 50=-5.87,1 \mathrm{~g} \mathrm{TGI} \mathrm{=} \mathrm{-5.54,}$

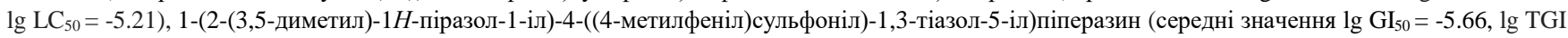
$\left.=-5.26, \lg \mathrm{LC}_{50}=-4.83\right)$ та 1-(2,4-біс((4-метилфеніл)сульфоніл)-1,3-тіазол-5-іл)піперазин (середні значення $1 \mathrm{~g}$ GI $50=-5.67$, $\lg$ TGI = -5.21, $\left.\lg \mathrm{LC}_{50}=-4.67\right)$ виявили найвищу інгібуючу активність. Отримані результати свідчать про перспективність пошуку серед ди- та трифункціональнозаміщених похідних 1,3-тіазолу нових протиракових препаратів.

Ключові слова: 1,3-тіазол; протиракова активність; інгібітори росту; цитостатична активність; цитотоксична активність. 